\title{
Enzymatic Preparation of Plasmonic-Fluorescent Quantum Dot-Gold Hybrid Nanoprobes for Sensitive Detection of Glucose and Alkaline Phosphatase and Dual- Modality Cell Imaging
}

Jie zhang, + Guohua $\mathrm{Qi},+\neq$ Chen $\mathrm{Xu},+\$$ and Yongdong Jin ${ }^{*}+\neq \rrbracket$

$\uparrow$ State Key Laboratory of Electroanalytical Chemistry, Changchun Institute of Applied Chemistry, Chinese Academy of Sciences, Changchun, Jilin 130022, P. R. China.

\#University of Chinese Academy of Sciences, Beijing 100049, P. R. China

§University of Science and Technology of China, Hefei 230026, P. R. China

*e-mail: ydjin@ciac.ac.cn

\section{Table of Contents:}

Figure S1. The PL intensities of polymer-coated QDs against $\mathrm{H}_{2} \mathrm{O}_{2}$ chemical oxidation and L-ascorbic acid etching.... S-2

Figure S2. The time evolution fluorescence emission spectra of the QD- enzyme solution after adding $\mathrm{HAuCl}_{4}$ solution. S-2

Figure S3. The time evolution fluorescence emission spectra of the QD- polymer-GOx solution in the presence of $\mathrm{HAuCl}_{4}$ after adding $20 \mu \mathrm{L}$ of glucose $\left(2 \times 10^{-3} \mathrm{M}\right)$.

Figure S4. Selectivity analysis for glucose detection by the PL intensity of the QD-GOx NSs

Figure S5. The fluorescence emission spectra of the QD-polymer-ALP solution after adding different concentrations of AAP in PBS.

Figure S6. The fluorescence intensity of the ALP sensing system as a function of the $\mathrm{pH}$ values.

Figure S7. The fluorescence intensity of the ALP sensing system as a function of the enzymatic reaction time in the presence and absence of ALP

Figure S8. Representative TEM images and the core-shell separation distribution of the QD-gold coreshell nanoparticles

Figure S9. The representative XPS spectra of the QD-Au NPs prepared by ALP enzymatic reaction.

Figure S10. The photostability of the polymer-coated QD and QD-gold hybrid nanoparticles.... 

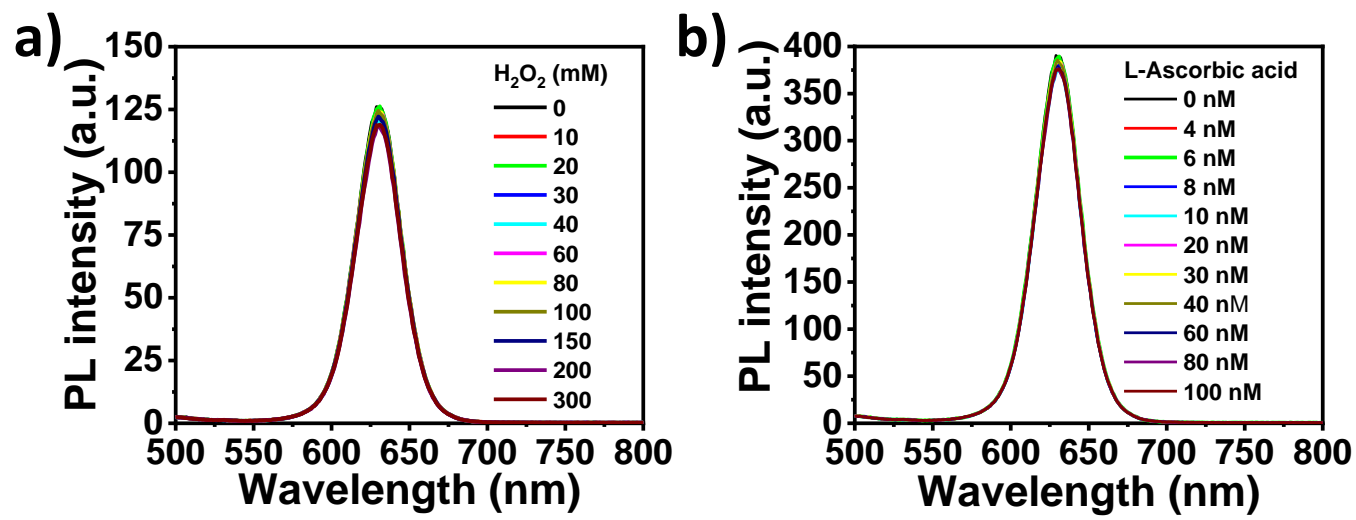

Figure S1. The PL intensities of polymer-coated QDs against chemical oxidation and L-ascorbic acid etching, in the presence of increasing concentrations of $\mathrm{H}_{2} \mathrm{O}_{2}$ and L-ascorbic acid.
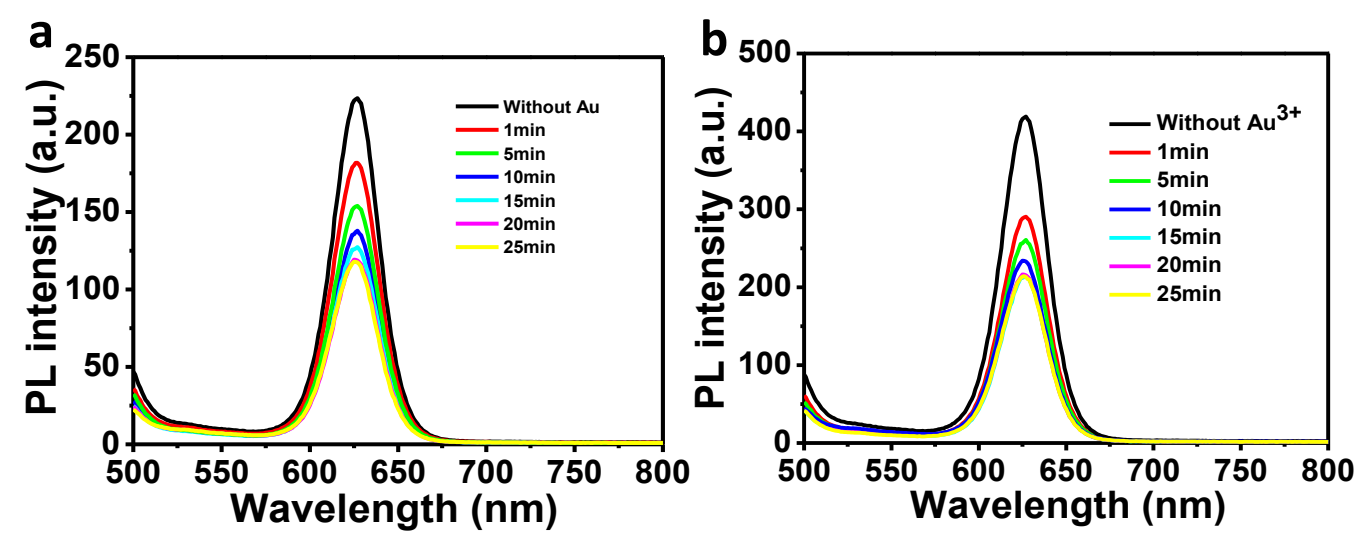

Figure S2. The time evolution fluorescence emission spectra of the QD- polymer-GOx and QD-polymerAAP solution after adding $\mathrm{HAuCl}_{4}$ solution $(10 \mu \mathrm{L}$, w/w $1 \%)$. 


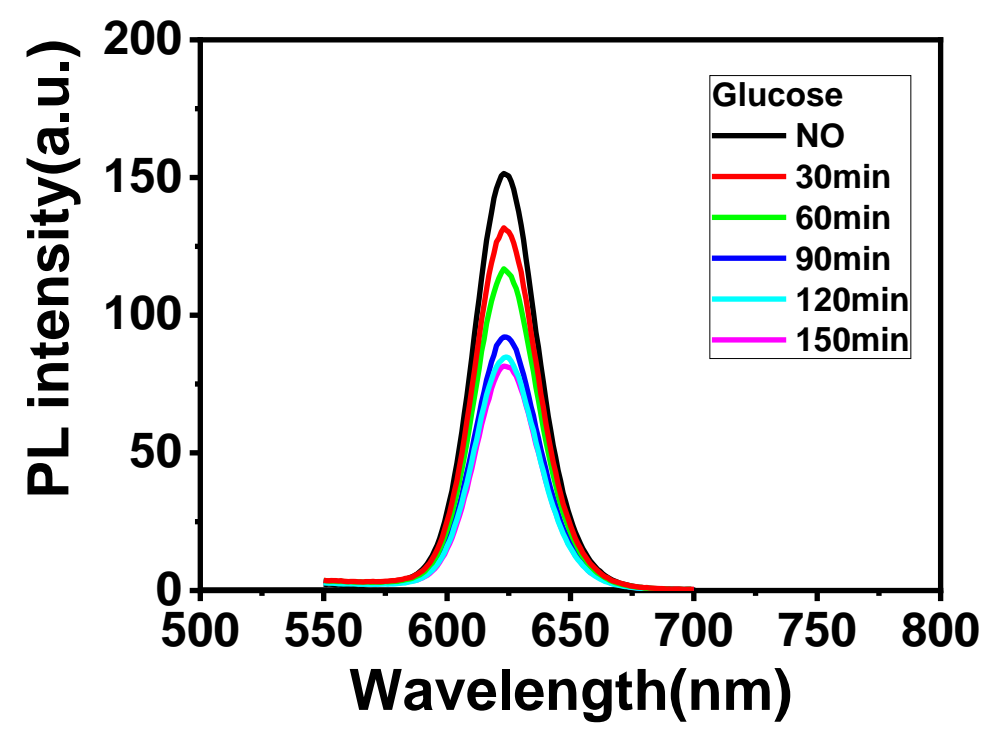

Figure S3. The time evolution fluorescence emission spectra of the QD- polymer-GOx solution in the presence of $\mathrm{HAuCl}_{4}$ after adding $20 \mu \mathrm{L}$ of glucose $\left(2 \times 10^{-3} \mathrm{M}\right)$.

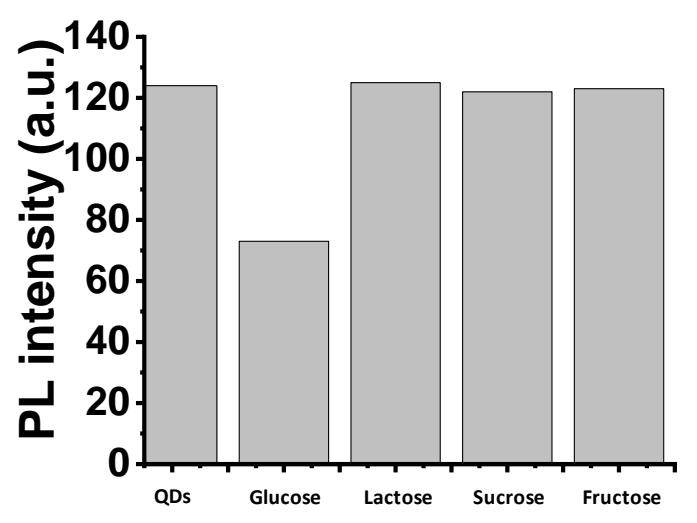

Figure S4. Selectivity analysis for glucose detection by the PL intensity of the QD-GOx NSs after $2 \mathrm{~h}$ incubation with glucose $\left(2 \times 10^{-3} \mathrm{M}\right)$, lactose $\left(4 \times 10^{-3} \mathrm{M}\right)$, sucrose $\left(4 \times 10^{-3} \mathrm{M}\right)$, and fructose $\left(4 \times 10^{-3} \mathrm{M}\right)$, respectively. 


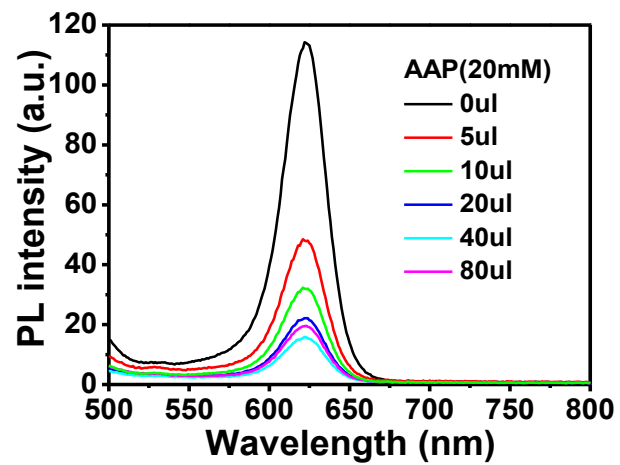

Figure S5. The fluorescence emission spectra of the QD-polymer-ALP solution after adding different concentrations of AAP in PBS (pH 7.4, $10 \mathrm{mM}$ ).

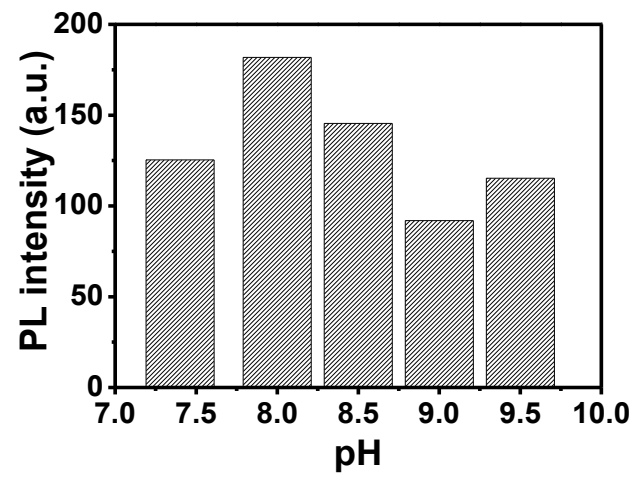

Figure S6. The fluorescence intensity of the ALP sensing system as a function of the pH values (10 mM Tris- $\mathrm{HCl})$. ALP, $0.05 \mathrm{mU} / \mathrm{mL}$; incubation at room temperature $\left(25-30^{\circ} \mathrm{C}\right)$ for $30 \mathrm{~min}$. $\lambda \mathrm{ex}=450 \mathrm{~nm}$. 


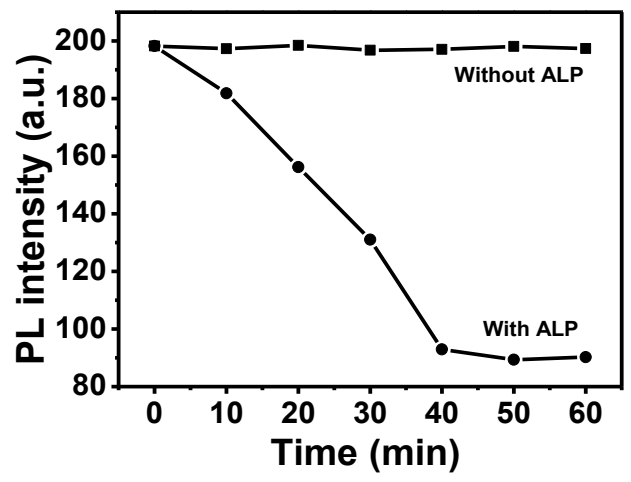

Figure S7. The fluorescence intensity of the ALP sensing system as a function of the enzymatic reaction time in the presence and absence of ALP, $0.05 \mathrm{mU} / \mathrm{mL}$; $\mathrm{pH} 9.0(10 \mathrm{mM}$ Tris- $\mathrm{HCl})$; incubation at room temperature $\left(25-30^{\circ} \mathrm{C}\right) . \lambda \mathrm{ex}=450 \mathrm{~nm}$.
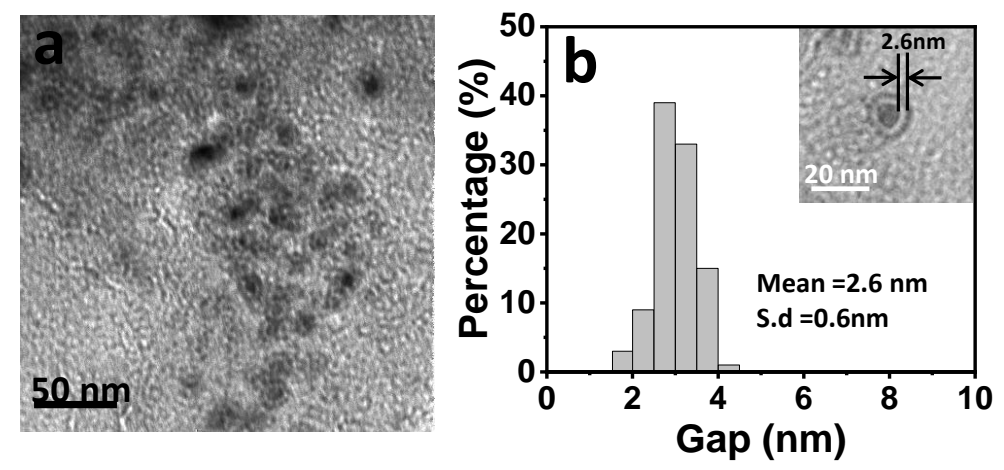

Figure S8. a) Representative TEM images of the QD-gold core-shell nanoparticles with layers of polyelectrolyte spacers. b) Histograms of the core-shell separation distribution of particles with polyelectrolytes layers. Each histogram was plotted based on >100 nanoparticles. Insets show the TEM measurements of representative particles. 

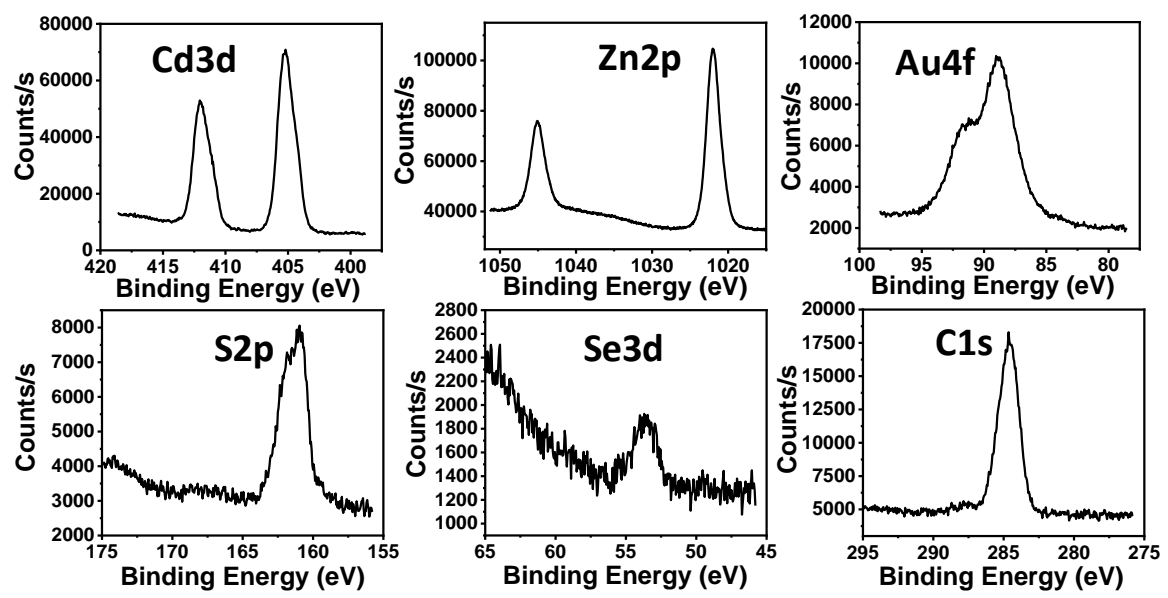

Figure S9. The representative XPS spectra of the QD-Au NPs prepared by ALP enzymatic reaction.
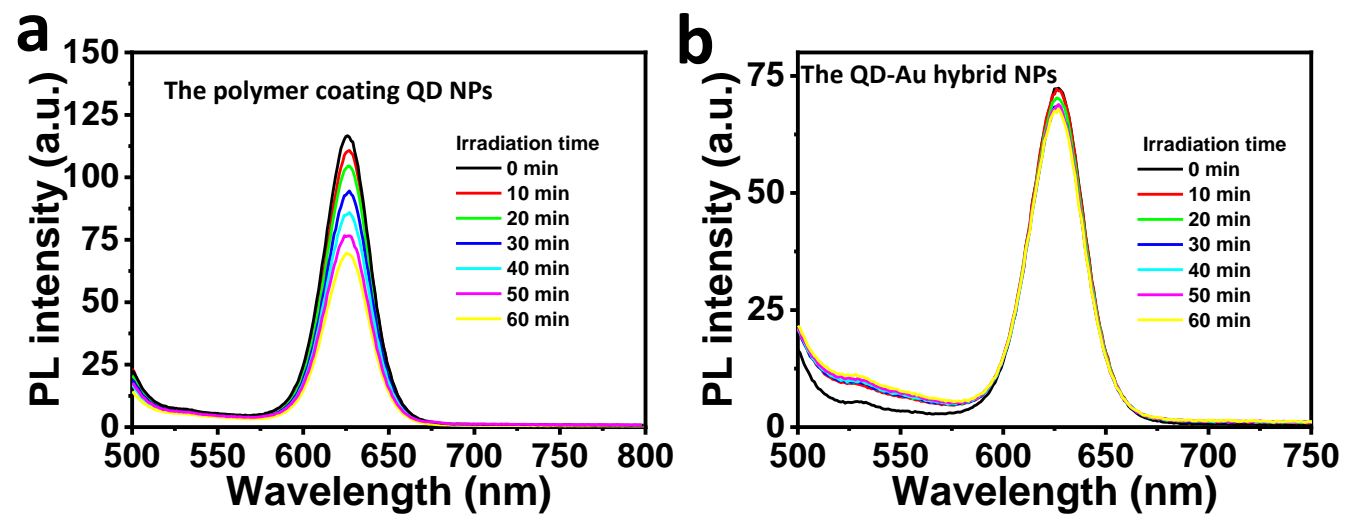

Figure S10. The fluorescence intensities of water-soluble polymer-coated QDs and QD-Au core-shell NPs. Under identical illumination conditions (450 $\mathrm{nm}$ laser). 